\title{
FATORES DE RISCO PARA O DESENVOLVIMENTO DE HIPERTENSÃO ARTERIAL ENTRE MOTORISTAS CAMINHONEIROS
}

Helisamara Mota Guedes ${ }^{1}$, Kátia de Arêdes Brum², Patrícia Andrade Costa ${ }^{2}$, Martha Elisa Ferreira de Almeida ${ }^{3}$

\begin{abstract}
RESUMO: Pesquisa descritiva-exploratória, de delineamento transversal, que teve como objetivo identificar o perfil dos motoristas caminhoneiros que trafegam na BR-381 quanto aos fatores de riscos modificáveis e não modificáveis para o desenvolvimento da hipertensão arterial. Utilizou-se instrumento para coleta e registro de dados demográficos, fatores de risco e níveis pressóricos de 257 motoristas; os dados foram tabulados e expressos em frequência absoluta e percentagem. A faixa etária variou entre 30 a 39 anos; 54,1\% relataram possuir parentes diretos hipertensos; 49,4\% e 23,7\% relataram uso bebida alcoólica e tabaco, respectivamente. Em relação aos hábitos alimentares, 39,3\% informaram ingerir alimentos processados e in natura e 13,2\% alimentos fritos; 26,8\% relataram acrescentar sal à alimentação depois de preparada. Os fatores de risco identificados podem favorecer o desenvolvimento da hipertensão arterial, fazendo-se necessária a realização de ações de saúde para esse grupo da população.
\end{abstract}

PALAVRAS-CHAVE: Hipertensão; Fatores de risco; Educação em saúde.

\section{RISK FACTORS FOR HYPERTENSION AMONG TRUCK DRIVERS}

\begin{abstract}
It is a descriptive, exploratory and transversal research which aimed to identify the profile of truck drivers that travel on the BR-381 regarding the modifiable risk factors and non-modifiable for the development of hypertension. An research instrument was used to collect and record demographics data, risk factors and blood pressure levels of 257 drivers. Then, data was tabulated and expressed in percentage and absolute frequency. Their ages ranged from 30 to 39 years, $54.1 \%$ reported having relatives working hypertension; $49.4 \%$ and $23.7 \%$ reported using alcohol and tobacco, respectively. Regarding eating habits, 39.3\% reported eating processed and fresh foods, $13.2 \%$ fried meals, and $26.8 \%$ reported adding salt to food after preparation. The risk factors identified may foster the development of hypertension, making it necessary to carry out health actions for this population group.
\end{abstract}

KEYWORDS: Hypertension; Risk factors; Health education.

\section{FACTORES DE RIESGO PARA EL DESARROLLO DE HIPERTENSIÓN ARTERIAL ENTRE CONDUCTORES CAMIONEROS}

RESUMEN: Investigación descriptiva-exploratoria de delineación transversal que tuvo como objetivo identificar el perfil de los conductores camioneros que transitan en la BR-381 cuanto a los factores de riesgos cambiables y no cambiables para el desarrollo de la hipertensión arterial. Se ha utilizado como instrumento para recoger y registrar los datos demográficos, factores de riesgo y niveles de presión de 257 conductores; los datos fueron tabulados y expresos en frecuencia absoluta y porcentaje. La franja etaria ha variado entre 30 y 39 años; 54,1\% referieron poseer parientes directos hipertensos; 49,4\% y 23,7\% referieron uso de bebida alcohólica y tabaco, respectivamente. Acerca de los hábitos alimentares, 39,3\% informaron ingerir alimentos procesados e in natura y 13,2\% alimentos freídos; $26,8 \%$ referieron acrecentar sal a la alimentacion depués de lista. Los factores de riesgo identificados pueden favorecer el desarrollo de la hipertensión arterial, haciendose necesaria la realización de acciones de salud para ese grupo de la población.

PALABRAS CLAVE: Hipertensión; Factores de riesgo; Educación en salud.

${ }^{1}$ Enfermeira. Mestre em Enfermagem. Professor Assistente da Universidade Federal do Vale do Jequitinhonha e Mucuri-UFVJ.

${ }^{2}$ Enfermeira do Centro Universitário do Leste de Minas Gerais.

${ }^{3}$ Nutricionista. Professor Assistente da Universidade Federal de Viçosa-UFV.

Autor correspondente:

Helisamara Mota Guedes

Universidade Federal do Vale do Jequitinhonha e Mucuri

Av. Jorge Dias Duarte, 214 - 35182-352 - Timóteo-MG-Brasil

Recebido: 30/03/10

E-mail: helisamaraguedes@yahoo.com.br

Aprovado: 10/11/10

Cogitare Enferm. 2010 Out/Dez; 15(4):652-8 


\section{INTRODUÇÃO}

O Brasil possui uma frota aproximada de 1,8 milhões de caminhões e uma população de mais de 700 mil motoristas caminhoneiros, composta quase que exclusivamente por homens que viajam uma média anual de 175 mil quilômetros ${ }^{(1)}$. O excesso de trabalho dessa categoria profissional tem causado vários problemas de saúde, entre os quais a perda auditiva induzida por ruído, estresse, câncer, doenças do sono, refluxo gastroesofágico, doenças cardiovasculares e músculo-esqueléticas e hipertensão arterial. As condições de trabalho como a alta carga, tráfego intenso, contato limitado com outros colegas e familiares, e o hábito de fumar têm sido considerado fatores importantes para o aumento dos risco de infarto do miocárdio $^{(2)}$.

A hipertensão arterial, por sua vez, é responsável pela alta frequência de internações e caracteriza-se pela elevação dos níveis pressóricos; pode gerar complicações como doenças cerebrovasculares, doença arterial coronariana, insuficiência cardíaca, insuficiência renal crônica e doença vascular periférica $^{(3-4)}$.

A doença pode ser controlada com tratamento não farmacológico que inclui a restrição de alimentos ricos em sódio, lipídios e carboidratos simples; abandono do tabagismo e do consumo de bebidas alcoólicas, controle do peso e do estresse, bem como a realização de atividade física ${ }^{(3)}$. Geralmente, a hipertensão arterial não se apresenta de forma isolada; pode estar associada a fatores como obesidade, dislipidemia, hiperglicemia, idade, sexo, fatores socioeconômicos, sedentarismo, consumo excessivo de sal ${ }^{(4)}$, consumo de álcool e ingestão de dieta com elevado valor calórico, consumida à beira da estrada ${ }^{(5)}$.

Pesquisas feitas com motoristas caminhoneiros têm mostrado que essa população apresenta-se exposta a diversos riscos que predispõem a doenças ${ }^{(1-2,5-7)}$. Estudo realizado na BR-381, com 107 motoristas caminhoneiros detectou que $64,35 \%$ eram adultos e apresentavam sobrepeso ou obesidade; entre os idosos, $83,33 \%$ estavam com sobrepeso, $89,72 \%$ eram sedentários, $19,63 \%$ hipertensos, $10,28 \%$ diabéticos, $53,27 \%$ eram ansiosos, $43,93 \%$ relataram ser etilistas, $29,91 \%$ eram tabagistas e, quanto à circunferência abdominal, $51 \%$ encontravam-se com risco aumentado para eventos cardiovasculares $^{(7)}$.
Os motoristas caminhoneiros apresentamse como uma clientela diferenciada no Sistema Único de Saúde, por não conseguir aderir às normas e rotinas das Unidades Básicas de Saúde (UBS), uma vez que o seu trabalho exige grande tempo fora de casa, não tendo oportunidade de frequentar as UBS. Somando-se a isto, apresentam horários irregulares para dormir, alimentar-se e descansar, surgindo para os profissionais de saúde o desafio de planejar estratégias e ações criativas que contemplem essa população com vistas à promoção da saúde e prevenção de doenças ${ }^{(6)}$.

Neste contexto, este estudo teve como objetivo identificar o perfil dos motoristas caminhoneiros que trafegam na BR-381 quanto aos fatores de risco não modificáveis e modificáveis para o desenvolvimento da hipertensão arterial.

\section{METODOLOGIA}

Trata-se de um estudo transversal, descritivo-exploratório, desenvolvido com uma amostra composta de 257 motoristas caminhoneiros. Esse profissional foi abordado de forma individual, logo após estacionar o veículo num posto de combustível da BR-381, cenário do estudo, depois de autorização formal pelo responsável pelo estabelecimento.

O critério de inclusão dos pesquisados foi ter um ano ou mais de exercício da profissão, para garantir uma maior adaptação nesta profissão. A seleção da amostra foi realizada de forma aleatória, ocorrendo a coleta de dados do dia 1 ao dia 12 de junho de 2008, das 8:00 às 17:00 horas.

Os participantes foram convidados para o estudo e aqueles que concordaram foram encaminhados a um local reservado para elucidação do estudo, assinatura do Termo de Consentimento Livre e Esclarecido (TCLE), com base na Resolução 196/96 ${ }^{(8)}$, e coleta dos dados. Esses foram obtidos por meio de entrevista, após um intervalo de tempo de repouso do respondente, de 5 a 10 minutos, e preenchimento de instrumento de coleta de dados estruturado e adaptado ${ }^{(5)}$ para identificar os fatores de risco não modificáveis para a hipertensão arterial - idade, parentes diretos com a hipertensão arterial, dislipidemia e diabetes mellitus; e fatores de risco modificáveis - etilismo, tabagismo e o consumo de alimentos processados e ricos em lipídios e sal. Após a entrevista, foram aferidos os níveis pressóricos por um único avaliador, utilizando es- 
fignomanômetro anaeroide devidamente calibrado e estetoscópio ${ }^{(4)}$.

Os dados foram tabulados, expressos em frequência absoluta e percentagem, e apresentados em tabelas. $\mathrm{O}$ anonimato na pesquisa foi acordado entre todos os participantes e os pesquisadores.

O estudo foi aprovado pelo Comitê de Ética em Pesquisa do Centro Universitário do Leste de Minas Gerais, sob parecer n. 13.58.08.

\section{RESULTADOS E DISCUSSÃO}

Quanto ao perfil sociodemográfico, observouse que a maioria dos pesquisados $(33,5 \%)$ tinha idade entre 30 e 39 anos e $59,9 \%$ possuíam o $1^{\circ}$ grau completo (Tabela 1). A média de idade dos pesquisados foi de 40 anos; $4,3 \%$ eram idosos, sendo este um importante fator de risco para as doenças cardiovasculares, principalmente as dislipidemias ${ }^{(4)}$.

Tabela 1 - Perfil sociodemográfico de motoristas caminhoneiros que trafegam na BR-381. Timóteo, 2008

\begin{tabular}{lcc}
\hline Variável & $\mathbf{n}$ & $\mathbf{\%}$ \\
\hline Idade (anos) & & \\
$22-29$ & 40 & 15,6 \\
$30-39$ & 86 & 33,5 \\
$40-49$ & 68 & 26,5 \\
$50-59$ & 52 & 20,2 \\
$\geq 60$ & 11 & 4,3 \\
Escolaridade & & \\
Sem educação formal & 31 & 12,1 \\
$1^{\circ}$ grau completo & 154 & 59,9 \\
$2^{\circ}$ grau completo & 68 & 26,5 \\
$3^{\circ}$ grau incompleto & 4 & 1,6 \\
\hline
\end{tabular}

Foi observado que a maioria dos motoristas possuía apenas o primeiro grau completo, sugerindo que o nível de escolaridade pode impactar diretamente sobre os hábitos de saúde do indivíduo ${ }^{(9-10)}$. Todos os pesquisados eram do sexo masculino, entretanto, em estudo realizado na BR-153, foi observado que $0,8 \%$ era do sexo feminino ${ }^{(1)}$.

Ao realizar a avaliação, observou-se que 20,7\% possuíam níveis pressóricos característicos para o quadro de hipertensão arterial (Tabela 2). No estudo realizado na BR- $040^{(11)}$ foi observado que 53\% dos caminhoneiros apresentaram níveis pressóricos correspondendo ao quadro limítrofe e potencialmente ou sugestivo de hipertensão arterial.
Tabela 2 - Níveis pressóricos dos motoristas que trafegam na BR-381. Timóteo, 2008

\begin{tabular}{lccc}
\hline $\begin{array}{l}\text { Pressão arterial } \\
\text { sistólica/diastólica } \\
(\mathbf{m m H g})\end{array}$ & Classificação & n & $\mathbf{\%}$ \\
\hline$<120 /<80$ & Ótima & 112 & 43.6 \\
$<130 /<85$ & Normal & 55 & 21,4 \\
$130-139 / 85-89$ & Limítrofe & 37 & 14,4 \\
$140-159 / 90-99$ & $\begin{array}{c}\text { Hipertensão } \\
\text { estágio 1 }\end{array}$ & 47 & 18,3 \\
$160-179 / 100-109$ & $\begin{array}{c}\text { Hipertensão } \\
\text { estágio 2 }\end{array}$ & 3 & 1,2 \\
$\geq 180 / \geq 110$ & $\begin{array}{c}\text { Hipertensão } \\
\text { estágio 3 }\end{array}$ & 1 & 0,4 \\
$\geq 140 /<90$ & $\begin{array}{c}\text { Hipertensão } \\
\text { sistólica isolada }\end{array}$ & 2 & 0,8 \\
\hline
\end{tabular}

Questionados sobre hipertensão arterial, 18,7\% relataram possuir tal patologia, sendo que $33,4 \%$ desses não faziam o tratamento medicamentoso (Tabela 3 ). Dos $38,9 \%$ dos pesquisados que tinham o hábito de aferir a pressão arterial, $34 \%$ realizavam tal procedimento em ambiente domiciliar. Em outro estudo realizado na BR381, dos 107 motoristas estudados, 19,63\% relataram ter hipertensão arterial, 48,60\% não eram hipertensos e $31,77 \%$ desconheciam ter a patologia ${ }^{(7)}$.

Tabela 3 - Hipertensão arterial, tratamento medicamentoso e aferição da pressão arterial dos motoristas que trafegam na BR-381. Timóteo, 2008

\begin{tabular}{lcc}
\hline Variável & n & \% \\
\hline Hipertensão arterial & 48 & 18,7 \\
Sim & 189 & 73,5 \\
Não & 20 & 7,8 \\
Não sabe & & \\
Hipertensos que fazem tratamento & & \\
medicamentoso & 32 & 66,6 \\
Sim & 16 & 33,4 \\
Não & \\
Hábito de aferir a pressão arterial & 100 & 38,9 \\
Sim & 157 & 61,1 \\
Não & & \\
Local de aferição da pressão arterial & \multicolumn{2}{|c}{} \\
Farmácia & 44 & 44,0 \\
Domić́lio & 34 & 34,0 \\
Unidade Básica de Saúde & 14 & 14,0 \\
Outros & 8 & 8,0 \\
\hline
\end{tabular}


Quanto à ocorrência de hipertensão arterial nos familiares dos entrevistados, $54,1 \%$ relataram possuir parentes com essa patologia, $38,1 \%$ possuíam parentes com diabetes mellitus e $42,0 \%$ relataram ter familiares com um quadro de hipercolesterolemia (Tabela 4). Pode ser observado que $27,1 \%$ relataram que o pai era hipertenso e que $38,1 \%$ das mães apresentavam tal patologia, entretanto, não se deve associar a hipertensão arterial somente à hereditariedade, pois os hábitos de vida estão mais ligados diretamente a este fator, e contribuem para o surgimento da hipertensão arterial ou o seu agravamento.

Tabela 4 - Presença de patologias entre os familiares dos motoristas que trafegam na BR-381. Timóteo, 2008*

\begin{tabular}{|c|c|c|c|c|c|c|c|c|c|c|c|c|c|c|}
\hline Patologia & \multicolumn{2}{|c|}{ Total } & \multicolumn{2}{|c|}{ Pai } & \multicolumn{2}{|c|}{ Mãe } & \multicolumn{2}{|c|}{ Avós } & \multicolumn{2}{|c|}{ Irmãos } & \multicolumn{2}{|c|}{ Tios } & \multicolumn{2}{|c|}{ Outros } \\
\hline Sim & 139 & 54,1 & 59 & 27,1 & 83 & 38,1 & 20 & 9,2 & 27 & 12,4 & 25 & 11,5 & 4 & 1,8 \\
\hline Não & 118 & 46,9 & & & & & & & & & & & & \\
\hline \multicolumn{15}{|c|}{ Infarto Agudo do Miocárdio } \\
\hline Sim & 76 & 29,6 & 32 & 41,6 & 10 & 13,0 & 12 & 15,6 & 9 & 11,7 & 12 & 15,6 & 2 & 2,6 \\
\hline Não & 181 & 70,4 & & & & & & & & & & & & \\
\hline \multicolumn{15}{|c|}{ Acidente Vascular Encefálico } \\
\hline Sim & 48 & 18,7 & 18 & 36,7 & 4 & 8,2 & 10 & 20,4 & 4 & 8,2 & 11 & 22,4 & 2 & 4,1 \\
\hline Não & 209 & 81,3 & & & & & & & & & & & & \\
\hline \multicolumn{15}{|c|}{ Diabetes mellitus } \\
\hline Sim & 98 & 38,1 & 11 & 9,6 & 43 & 37,7 & 18 & 15,8 & 10 & 8,8 & 20 & 17,5 & 12 & 10,6 \\
\hline Não & 159 & 61,9 & & & & & & & & & & & & \\
\hline \multicolumn{15}{|c|}{ Hipercolesterolemia } \\
\hline Sim & 108 & 42,0 & 15 & 12,8 & 33 & 28,2 & 18 & 15,4 & 14 & 12,0 & 20 & 17,1 & 17 & 14,5 \\
\hline Não & 149 & 58,0 & & & & & & & & & & & & \\
\hline
\end{tabular}

*Os participantes responderam mais de uma alternativa quanto aos antecedentes familiares

$\mathrm{O}$ consumo frequente de medicamentos para inibir o sono foi relatado por $49,4 \%$ dos entrevistados, sendo que $33,1 \%$ relataram dirigir cansados; $30,0 \%$ relataram que algumas vezes perdem a atenção; $65,4 \%$ viajam de 400 a $899 \mathrm{~km} / \mathrm{dia}$; e $5,4 \%$ permanecem mais de 31 dias viajando sem retornar ao domicílio (Tabela 5). A quilometragem rodada acima ou igual a $900 \mathrm{~km} /$ dia foi relatada por $17,9 \%$ dos pesquisados, o que coloca esses trabalhadores em um grupo de risco para o desenvolvimento de doenças relacionadas ao estresse, como hipertensão arterial e dislipidemias ${ }^{(12)}$.

$\mathrm{O}$ uso de medicamentos para inibir o sono foi relatado por $57 \%$ dos caminhoneiros da BR-116 ${ }^{(5)}$, em $30 \%$ nos caminhoneiros da BR-153 ${ }^{(1)}$ e em $26,15 \%$ nos caminhoneiros da BR-381 ${ }^{(6)}$. Entretanto, em outro estudo, apenas $0,25 \%$ relataram consumir sempre tais medicações e $92,5 \%$ relataram nunca ter utilizado ${ }^{(2)}$. Dos avaliados na rodovia MG- $050^{(13)}$, verificou-se que $66 \%$ costumavam fazer uso de anfetaminas durante o percurso da viagem, sendo que $27 \%$ usavam diariamente e $60 \%$ entre duas a três vezes por semana. Deve ser destacado que seu uso por até três vezes na semana pode desencadear o desenvolvimento da dependência; a perda de horas de sono, associada ou não ao uso de substâncias psicoativas ${ }^{(14)}$. Ainda, pode implicar em falhas e erros nas atividades profissionais, favorecendo maior incidência de acidentes ${ }^{(6,15)}$. Geralmente, os profissionais recorrem aos inibidores de sono pela necessidade de percorrer grandes distâncias no dia e pela própria exigência do serviço, visto que muitos deles possuem horário para carregar e descarregar, enquanto outros ganham pela carga transportada ${ }^{(2,5-6)}$.

O hábito de dirigir cansado foi relatado por $30 \%$ dos motoristas da BR- $040^{(11)}$ e $35 \%$ dos motoristas da BR-116 ${ }^{(5)}$, o que pode trazer sérias consequências, como a maior probabilidade de ocorrência de acidentes no trânsito por falha humana, além de ser fator de risco para o desenvolvimento de hipertensão arterial. Caminhoneiros que trafegam regularmente por longas rotas, com no mínimo mil $\mathrm{km} / \mathrm{dia}^{(1)}$, possivelmente realizam esse trajeto em jornada de trabalho superior a 8 horas, sendo que acima desta carga horária há comprometimento do sono e do descanso, com implicações para a saúde física e mental, principalmente quanto às doenças cardiovasculares $^{(2)}$.

O consumo de bebidas alcoólicas foi relatado por $49,4 \%$ dos pesquisados e o tabaco por $23,7 \%$. Em 
estudo feito nesta mesma BR-381 ${ }^{(7)}$ foi observado que $43,93 \%$ eram etilistas e $29,91 \%$ eram tabagistas. O consumo frequente de álcool e tabaco foi relatado por $51 \%{ }^{(11)}$ e de $55 \%{ }^{(5)}$ respectivamente em estudos distintos. Entretanto, apenas 4\% e 21,25\% relataram fazer uso de bebidas alcoólicas e de tabaco sempre, enquanto $56,5 \%$ e $67,5 \%$ relataram nunca ter utilizado ${ }^{(2)}$. O uso de bebida alcoólica durante a jornada de trabalho foi relatado por $91 \%$ dos avaliados ${ }^{(13)}$, dos quais $24 \%$ utilizavam álcool todos os dias e $35 \%$ consumiam de duas a três vezes por semana, com a justificativa de participação na roda de amigos. Tem sido observado que os motoristas brasileiros apresentam um grande consumo de cerveja e aguardente ${ }^{(14)}$. Além de ser um dos principais fatores relacionados a causas de acidentes $^{(4,13)}$, a ingestão de álcool está diretamente relacionada ao desenvolvimento de hipertensão arterial. A redução do consumo de etanol diminui os níveis pressóricos sistólicos em 3,3 $\mathrm{mmHg}(2,5 \mathrm{a} 4,1 \mathrm{mmHg})$ e diastólicos em 2,0 mmHg (1,5 a 2,6 mmHg), sendo que o etanol ingerido fora do período das refeições propicia o desenvolvimento de hipertensão arterial, independentemente do volume ingerido ${ }^{(4)}$.

Tabela 5 - Hábitos relacionados à profissão, etilismo, tabagismo e consumo alimentar dos motoristas que trafegam na BR-381. Timóteo, 2008

\begin{tabular}{lcc}
\hline Variável & n & \% \\
\hline $\begin{array}{l}\text { Consumo de medicamento para inibir } \\
\text { o sono }\end{array}$ & & \\
Algumas vezes & 127 & 49,4 \\
Raramente & 30 & 11,7 \\
Nunca & 100 & 38,9 \\
Dirige cansado & & \\
Frequentemente & 85 & 33,1 \\
Algumas Vezes & 84 & 32,7 \\
Raramente & 60 & 23,3 \\
Nunca & 28 & 10,9 \\
Perde a concentração & & \\
Frequentemente & 50 & 19,5 \\
Algumas Vezes & 77 & 30,0 \\
Raramente & 57 & 22,2 \\
Nunca & 73 & 28,3 \\
Distância percorrida (Km/dia) & & \\
100 - 399 & 43 & 16,7 \\
400 - 899 & 168 & 65,4 \\
900 - 1299 & 40 & 15,6 \\
$\geq 1300$ & 6 & 2,3 \\
\hline
\end{tabular}

Continua

\begin{tabular}{lcc}
\hline Permanência de dias viajando (dias) & & \\
$0-10$ & 181 & 70,4 \\
$11-20$ & 46 & 17,9 \\
$21-30$ & 16 & 6,2 \\
$31-40$ & 6 & 2,3 \\
$\geq 41$ & 8 & 3,1 \\
Etilismo & & \\
Sim & 127 & 49,4 \\
Não & 88 & 34,3 \\
Parou & 42 & 16,3 \\
Tabagismo & & \\
Sim & 61 & 23,7 \\
Não & 150 & 58,4 \\
Parou & 46 & 17,9 \\
Preferência de consumo de alimentos & & \\
In natura & 96 & 37,4 \\
Processados & 60 & 23,3 \\
Ambos & 101 & 39,3 \\
Forma preferencial de preparo & & \\
Cozido & 82 & 31,9 \\
Assado & 64 & 24,9 \\
Fritos & 34 & 13,2 \\
Grelhados & 9 & 3,5 \\
Todos & 68 & 26,5 \\
Acrescenta sal aos alimentos depois & & \\
de preparados & & \\
Sim & 69 & 26,8 \\
Não & 188 & 73,2 \\
Usa sal nos temperos & & \\
Sim & 161 & 62,6 \\
Não & 96 & 37,4 \\
\hline & & \\
\hline
\end{tabular}

Foi observada resistência dos participantes para responder sobre o consumo de etanol, devendo ser destacado que a coleta de dados coincidiu com a aprovação da Lei $11.705 / 08$, que proibia a ingestão de qualquer quantidade de álcool por condutores, conhecida popularmente por "lei seca". Depois da implantação dessa lei observou-se que a taxa de acidentes diminuiu em $30 \%$ no mês de julho e nos primeiros 15 dias de agosto de 2008 , em relação ao mesmo período de $2007^{(16)}$.

O uso do tabaco é um fator de risco evitável para o desenvolvimento da hipertensão arterial e suas complicações, pois a nicotina promove a vasoconstrição, aumentando, consequentemente, a pressão arterial. Interromper o uso do cigarro é extremamente importante para evitar essa patologia, assim como valorizar a realização de atividades físicas e a reeducação alimentar ${ }^{(17)}$. 
Em relação aos hábitos alimentares, 39,3\% dos avaliados relataram ingerir tanto alimentos processados quanto in natura e $13,2 \%$ preferiam alimentos fritos. Em outro estudo foi observado que 33,25\% dos avaliados relataram sempre consumir alimentação gordurosa, enquanto $15,5 \%$ relataram nunca consumir tais alimentos ${ }^{(2)}$.

O controle da alimentação é importante para a prevenção da hipertensão arterial, principalmente quanto às fontes lipídicas, que devem ser reduzidas ou evitadas ${ }^{(4)}$. Em uma pesquisa realizada na BR-381, observou-se que $46,73 \%$ dos entrevistados relataram que faziam três refeições diárias (desjejum, almoço e jantar); $88,78 \%$ disseram que faziam suas refeições em restaurantes, sendo que $77,57 \%$ não as realizavam em horários habituais. Foi alegado por estes que havia um tempo previsto para a entrega de cargas e que, muitas vezes, não paravam para realizar suas refeições devido à falta de tempo e por saber que os horários de funcionamentos dos restaurantes em postos de gasolina eram ampliados ${ }^{(7)}$.

No presente estudo, observou-se que $26,8 \%$ dos entrevistados relataram acrescentar sal à alimentação depois de preparada, fato este que pode ser uma consequência do acesso direto aos saleiros nos restaurantes, o que deve ser desestimulado, pois acarreta danos à saúde. Uma redução do consumo de sal pode acarretar a diminuição de até $20 \%$ nos níveis pressóricos $^{(4)}$.

Quanto à opinião dos caminhoneiros em relação às ações dos profissionais de saúde que poderiam auxiliar na melhoria da qualidade de vida dos mesmos, observou-se que $63,8 \%$ não souberam responder a pergunta, $31,1 \%$ sugeriram atendimento em postos de gasolina de grande fluxo de motoristas e $3,1 \%$ sugeriram a realização de campanhas educativas sobre a hipertensão arterial. Visando uma melhoria da qualidade de vida do grupo estudado, a realização de campanhas educativas sobre a hipertensão arterial é importante, uma vez que o enfermeiro tem um papel fundamental como educador em saúde, pois proporciona ao paciente hipertenso o conhecimento sobre sua doença e tratamento adequado ${ }^{(3)}$.

\section{CONCLUSÃo}

Os principais fatores de risco não modificáveis observados foram a idade e a presença de parentes diretos com a hipertensão arterial, diabetes mellitus e hipercolesterolemia. Os principais fatores modificáveis foram o etilismo, tabagismo, consumo de alimentos processados e ricos em lipídios e o acréscimo de sal à alimentação depois de preparada. Conclui-se que se faz necessária a realização de ações de saúde para estes motoristas, principalmente em postos de gasolina de grande fluxo, pois os mesmos alegaram não ter disponibilidade para buscar o serviço de saúde para a prevenção e/ou tratamento da hipertensão arterial.

\section{REFERÊNCIAS}

1. Teles SA, Matos MA, Caetano KAA, Costa LA, França DDS, Pessoni GC, et al. Comportamentos de risco para doenças sexualmente transmissíveis em caminhoneiros no Brasil. Rev Panam Salud Pública. 2008;24(1):25-30.

2. Penteado RZ, Gonçalves CGO, Costa DD, Marques JM. Trabalho e saúde em motoristas de caminhão no interior de São Paulo. Saude Soc. 2008;17(4):35-45.

3. Severo DF, Amestoy SC, Thofehrn MB, Goldmeier S. Conhecimento e modificações de comportamento frente ao tratamento não-farmacológico da HAS: antes e após educação em saúde do profissional enfermeiro. Cogitare Enferm. 2009;14(3):506-11.

4. V Diretrizes Brasileiras de Hipertensão Arterial - Sociedade Brasileira de Cardiologia, Sociedade Brasileira de Hipertensão, Sociedade Brasileira de Nefrologia 2006 (acesso em 30 jun 2009). Disponível: http://tiny.cc/jrdhs

5. Cavagioni LC. Perfil dos riscos cardiovasculares em motoristas profissionais de transportes de carga da Rodovia BR - 116 no trecho Paulista-Régis Bittencourt [dissertação]. São Paulo (SP): Universidade de São Paulo; 2006.

6. Botelho SSP, Guedes HM, Paes MSL. Uso do "rebite" entre caminhoneiros que trafegam na BR-381 do interior do estado de Minas Gerais. Enferm Bras. 2008;7(3):134-40.

7. Martins EPA, Silva AS, Guedes HM. Fatores de risco para obesidade entre caminhoneiros que trafegam na BR 381. Rev Enferm Integrada. 2009;2(2):345-57.

8. Ministério da Saúde (BR). Conselho Nacional de Saúde. Diretrizes e normas regulamentadoras de pesquisa envolvendo seres humanos. Resolução n. 196, de 10 de outubro de 1996. Brasília; 1996. 
9. Villarinho L, Bezerra I, Lacerda R, Latorre MRDO, Paiva V, Stall R, et al. Caminhoneiros de rota curta e sua vulnerabilidade ao HIV, Santos, SP. Rev Saúde Pública. 2002;36 Suppl 4: S62-7.

10. Nascimento E. Desenvolvimento de pesquisa-ação com motoristas de caminhão de estrada: trabalhando na problematização das questões voltadas a sexualidade: DST/AIDS e remédios [tese]. Ribeirão Preto (SP): Universidade de São Paulo; 2003.

11. Batista MAS, Silva FAB. Nível de saúde de caminhoneiros que trafegam pela BR 040 , com base em dados obtidos durante o VI comando Rodoviário Federal na cidade de Brasília-DF: um estudo de caso. Revista de Divulgação Científica da FACESA [Internet] 2005 [acesso em 30 jun 2009]. Disponível: http://www.senaaires.com.br/revistavirtual/artigos/ ArtCient/ArtCient1.pdf

12. Luzia RWS, Almeida MEF. Perfil dos professores de um curso de enfermagem quanto a seus níveis pressóricos, estresse e de qualidade de vida. Enferm Bras. 2010;9(1):15-21.

13. Nascimento EC, Nascimento E, Silva JP. Uso de álcool e anfetaminas entre caminhoneiros de estrada. Rev Saúde Pública. 2007;41(2):290-3.

14. Souza JC, Paiva T, Reimão R. Sono, qualidade de vida e caminhoneiros brasileiros e portugueses. Psicol Estud. 2008;13(3):429-36.

15. Canani SF, Barreto SSM. Sonolência e acidentes automobilísticos. J Pneumol. 2001;27(2):94-6.

16. Moraes AE. Frente parlamentar em defesa do trânsito seguro. Lei salva vidas. Câmara dos Deputados: 2008. [acesso em 30 jun 2009]. Disponível: http://tiny.cc/ vichr

17. Araújo AJ, Menezes AMB, Dórea AJPS, Torres BS, Viegas CAS, Silva CAR, et al. Diretrizes para cessação do tabagismo. J Bras Pneumol. 2004;30(2):1S-76S. 